\title{
A Label Correcting Algorithm with Storing Partial Solutions to Solving the Bus Routing Problem
}

\author{
Jacek WIDUCH \\ Silesian University of Technology, Institute of Informatics \\ ul. Akademicka 16, 44-100 Gliwice, Poland \\ e-mail: jacek.widuch@polsl.pl
}

Received: February 2012; accepted: December 2012

\begin{abstract}
In the paper we describe the bus routing problem (BRP), which the goal is to find a route from the start stop to the final stop minimizing the time and the cost of travel and the length of the route. Additionally the time of starting travel at the start stop is given. Analysis of the problem is presented and in particular we point at properties of routes. The BRP is an example of multicriteria optimization problem (MOP), which the solution is the set of non-dominated solutions. This paper proposes a label correcting algorithm with storing partial solutions for solving the BRP. The algorithm makes possible to find all routes which belong to the set of non-dominated solutions. Apart from that the results of experimental tests are presented. Additionally the results are compared with results for the BRP where the goal is to minimize only the time and the cost of travel and the length of the route is not taken into consideration.

Key words: bus routing problem, multicriteria optimization, set of non-dominated solutions, multicriteria shortest path problem, directed weighted graph, variable weights, shortest path, loopless path, label correcting algorithm.
\end{abstract}

\section{Introduction}

The shortest path problem is among the most studied the graph optimization problems. Given a graph with a single weight function, the goal is to find a path with the minimal weight. It has been the subject of extensive research for many years resulting in the publication of a large number of papers. There are well known algorithms for finding the shortest path proposed by Dijkstra (1959), Bellman (1958), Ford (1956), Floyd (1962), Warshall (1962) and Johnson (1977). In many cases using a single weight function is insufficient because it does not describe precisely the studied problem. In this case, a graph with $k(k>1)$ weight functions is used and the problem is called the multicriteria shortest path (MSP) problem.

The MSP problem is an example of multicriteria optimization and it is known to be NP-complete by transformation from a 0-1 knapsack problem (Garey and Johnson, 1990; Hansen, 1980; Skriver, 2000a). Many algorithms for solving the BSP problem are known and these algorithms are classified into the following categories: label setting algorithms (Hansen, 1980; Martins, 1984; Tung and Chew, 1988), label correcting algorithms (Brumbaugh-Smith and Shier, 1989; Corley and Moon, 1985; Daellenbach and 
De Kluyver, 1980; Skriver and Andersen, 2000b), $k$-th shortest path algorithms (Climaco and Martins, 1982), two phases algorithms (Mote et al., 1991) and the others (Dell'Olmo et al., 2005; Machuca et al., 2009; Mandow and Pérez-de-la-Cruz, 2008a; Mandow and érez-de-la-Cruz, 2008b; Martí et al., 2009; Raith and Ehrgott, 2009). All mentioned algorithms assume constant weights, i.e. the weight function has a constant value for a given arc of graph and it does not change. There are differences between problem with constant and variable weights.

In this paper the BRP, which is an example of the MSP problem with variable weights, is presented and analysed. The bus network is represented by the graph with weight functions. The weight functions determine the cost and the travel time and their values are calculated during the process of finding the solutions. For that reason, values of both weight functions are not constant for a given arc. In the paper we have made a theoretical analysis of the problem and with particular emphasis on differences between the problem with constant weights and the problem with variable weights. The algorithm for solving the BRP is also presented and it belongs to the group of label correcting algorithms with storing partial solutions. The algorithm was implemented and tested and the test results are also presented.

The paper is divided into five sections. Section 2 contains description of the BRP, i.e. a formulation of the BRP (Section 2.1) and an analysis of the BRP, in particular pointed at differences between the MSP with variable weights and constant weights (Section 2.2). The label correcting algorithm for solving the BRP is described in Section 3. In Section 4 experimental test results are reported. Conclusions are outlined in the final section.

\section{The Bus Routing Problem (BRP)}

\subsection{Formulation of the BRP}

The BRP is related to the choice of means of transport and finding the route of travel between the two given points. We have given the bus network consisted of $n$ stops $s_{1}, \ldots, s_{n}$. In the network buses of $M$ bus lines numbered from 1 to $M$ are run. Additionally, the network is divided into zones, which determines the cost of travel.

The route of the bus line consists of the sequence of stops through which the bus runs from the start stop to the final stop of the line and it is defined for each bus line. The travel between two given stops is directed. If the bus runs from stop $s_{i}$ to stop $s_{j}$, it does not imply that the bus of the same line runs in the opposite direction, i.e. from $s_{j}$ to $s_{i}$. The bus of a given bus line can run in both directions, but these routes can be different. The route of each bus line consists of different stops except for the start and the final stops which can be the same. If the final stop is the same as the start stop, then the bus line is called the circular line.

Let the route of $i$-th bus line $(i=1, \ldots, M)$ consists of sequence of stops:

$$
\left\langle s_{0}^{i}, s_{1}^{i}, \ldots, s_{k-1}^{i}, s_{k}^{i}\right\rangle
$$


where $s_{0}^{i}$ is the start stop and $s_{k}^{i}$ is the final stop of the line. The bus of $i$-th line runs between stops belonging to the route with the given frequency, i.e. it leaves the start $\operatorname{stop} s_{0}^{i}$ at time $T_{0}^{i}$, passes through stops $s_{1}^{i}, \ldots, s_{k-1}^{i}$ at times $T_{0}^{i}+\delta_{0}^{i}, \ldots, T_{0}^{i}+\delta_{k-2}^{i}$, respectively, and it reaches the final stop $s_{k}^{i}$ at time $T_{0}^{i}+\delta_{k-1}^{i}$. The next course the bus of this line starts at time $T_{1}^{i}\left(T_{1}^{i}=T_{0}^{i}+\beta_{0}^{i}\right)$ and thus it reaches stops $s_{1}^{i}, \ldots, s_{k}^{i}$ at times $T_{1}^{i}+\delta_{0}^{i}, \ldots, T_{1}^{i}+\delta_{k-1}^{i}$. The bus of $i$-th bus line executes $p^{i}$ courses and leaves the start stop $s_{0}^{i}$ at times $T_{0}^{i}, \ldots, T_{p-1}^{i}\left(T_{0}^{i}<\cdots<T_{p-1}^{i}\right)$, where $T_{j}^{i}=T_{0}^{i}+\beta_{j-1}^{i}(j=1, \ldots, p-1)$. The $T_{0}^{i}$, $\beta_{0}^{i}, \ldots, \beta_{p-2}^{i}, \delta_{0}^{i}, \ldots, \delta_{k-1}^{i}\left(0<\beta_{0}^{i}<\cdots<\beta_{p-2}^{i} ; 0<\delta_{0}^{i}<\cdots<\delta_{k-1}^{i}\right)$ values are defined by the timetable of the bus line. We omit the times of getting on and off the bus by passengers, i.e. we assume that the time of departure from a stop equals the time of arrival to this stop.

For defined the bus network structure, the bus lines routes and the timetable, we have given the start stop $s_{s}$ and the final stop $s_{e}\left(s_{s} \neq s_{e}\right)$ between which we want to travel. The time of starting travel $T_{S}$ at the start stop is given additionally. The objective is to find a route from $s_{S}$ to $s_{e}$ minimizing the time and the cost of travel and the length of the route. We should determine all stops belonging to the route, the bus lines which we travel between stops, stops of changes and times of departure from all stops belonging to the route.

The time of the travel depends on the chosen route and the possible stops of changes. It is the sum of the travel times between stops belonging to the route, ${ }^{1}$ time of waiting at the start stop and times of waiting for changes. The cost of travel depends on the location of the stops in the area of zones and it is calculated as follows. A ticket for a single travel (a travel without a bus change) within the area of a single zone equals $c_{1}\left(0<c_{1}\right)$ units, within two zones it equals $c_{2}\left(c_{1}<c_{2}\right)$ units and within the confines of more than two zones it equals $c_{3}\left(c_{2}<c_{3}\right)$ units. $^{2}$ For that reason the cost of travel from the start stop $s_{s}$ to the final stop $s_{e}$ is equal to the sum of costs of travel between the stops of bus changes. Among bus lines there are fast lines. The travel by bus of a fast line is faster than travel by a regular line which takes into account the timetable. The cost of the travel by a fast line is twice as large as the cost of the travel by a regular line. The length of the route equals the number of stops belonging to the route.

\subsection{Analysis of the BRP}

The bus network is described by the directed weighted graph $G=(V, E)$ with $|V|=n$ vertices $v_{1}, \ldots, v_{n}$ and $|E|=m$ arcs $e_{1}, \ldots, e_{m}\left(e_{j}=\left(v_{a}, v_{b}\right) ; v_{a}, v_{b} \in V\right)$ (Chartrand et al., 2010; Jungnickel, 1989; Wilson, 1996). The vertices represent the bus stops where the vertex $v_{j}$ represents the bus stop $s_{j}(j=1, \ldots, n)$. Thus a vertex expression with reference to the graph representing the bus network determines the bus stop of the network. The arc $e_{j}=\left(v_{a}, v_{b}\right)$ represents the route of travel of a specific bus line whose buses run

\footnotetext{
${ }^{1}$ The travel times between stops are defined by the timetable of bus lines.

${ }^{2}$ In a sample routes presented in the paper we assume the following costs of a ticket: $c_{1}=2.0, c_{2}=2.3$ and $c_{3}=2.6$ units.
} 


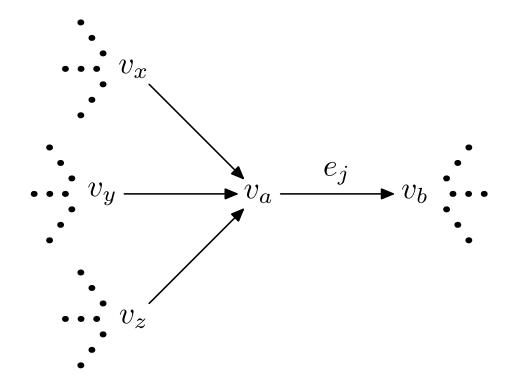

Fig. 1. A part of graph representing the bus network.

directly from a stop represented by $v_{a}$ to a stop represented by $v_{b}$. Direct travel denotes that the route from $v_{a}$ to $v_{b}$ does not include other vertices.

$$
\left\langle v_{0}^{i}, v_{1}^{i}, \ldots, v_{k-1}^{i}, v_{k}^{i}\right\rangle
$$

The route of $i$-th bus line descried by (1) is represented by a path (2) in the graph $G$ from the vertex $v_{0}^{i}$ to the vertex $v_{k}^{i}$, where $v_{0}^{i}$ represents the start stop $s_{0}^{i}$ and $v_{k}^{i}$ represents the final stop $s_{k}^{i}$ of the $i$-th bus line. The travel between two given stops is directed, thus if there exists the arc $\left(v_{a}, v_{b}\right)$, it does not imply that there exists the arc $\left(v_{b}, v_{a}\right)$. Between two given stops buses of many bus lines can run. For that reason the graph $G$ representing the bus network can contain parallel arcs. Each arc $e_{j}=\left(v_{a}, v_{b}\right)$ has three weights: $l\left(e_{j}\right)$, $t\left(e_{j}\right)$ and $c\left(e_{j}\right)$.

The weight $l\left(e_{j}\right)$ equals the line number the bus runs from $v_{a}$ to $v_{b}$ and it takes values from range $1, \ldots, M$. The weight $t\left(e_{j}\right)$ takes positive values, it is not constant and it equals $t\left(e_{j}\right)=T_{b}-T_{a}$, where $T_{b}$ is the time of arrival to $v_{b}$ and $T_{a}$ is the time of arrival to $v_{a}$. Thus it is a sum of the travel time from $v_{a}$ to $v_{b}$ and the possible time of waiting at $v_{a}$. The travel time from $v_{a}$ to $v_{b}$ is defined by the timetable and it is constant. The value of weight $t\left(e_{j}\right)$ is determined by the time of waiting at $v_{a}$ which is variable. Let us consider a part of the bus network presented in Fig. 1. There are many ways of travel to $v_{a}$ - in the first case we arrive from $v_{x}$ and the time of arrival to $v_{a}$ is $T_{a}^{x}$, in the second case we arrive from $v_{y}$ at $T_{a}^{y}$ and in the last case the time of arrival is $T_{a}^{z}$ and we arrive from $v_{z}$. Let us assume that the travel time from $v_{a}$ to $v_{b}$ equals $t_{a b}$ and the time of departure from $v_{a}$ to $v_{b}$ is the same in all cases and it equals $T_{a}$, but (3) is satisfied. The times of waiting at $v_{a}$ are equal to: $\Delta t_{a}^{x}=T_{a}-T_{a}^{x}, \Delta t_{a}^{y}=T_{a}-T_{a}^{y}$ and $\Delta t_{a}^{z}=T_{a}-T_{a}^{z}$. Since (3) is satisfied, so $\Delta t_{a}^{x} \neq \Delta t_{a}^{y} \neq \Delta t_{a}^{z}$ and the weight $t\left(e_{j}\right)$ in each case takes different values: $t^{\prime}\left(e_{j}\right)=\Delta t_{a}^{x}+t_{a b}, t^{\prime \prime}\left(e_{j}\right)=\Delta t_{a}^{y}+t_{a b}, t^{\prime \prime \prime}\left(e_{j}\right)=\Delta t_{a}^{z}+t_{a b}$. Therefore the weight $t\left(e_{j}\right)$ is not constant.

$$
T_{a}^{x} \neq T_{a}^{y} \neq T_{a}^{z}
$$

The weight $c\left(e_{j}\right)$ takes non-negative values and it equals $c\left(e_{j}\right)=c_{b}-c_{a}$, where $c_{b}$ equals the cost of travel to $v_{b}$ and $c_{a}$ equals the cost of travel to $v_{a}{ }^{3}$ The values $c_{a}$ and $c_{b}$

\footnotetext{
${ }^{3}$ We assume the travel by a bus of regular line, otherwise the value $c\left(e_{j}\right)$ must be multiplied by 2 .
} 


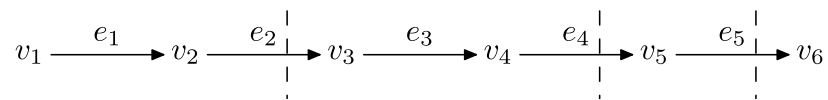

Fig. 2. The route of a bus line. The zone borders are denoted by the dashed lines.

Table 1

The values $c\left(e_{1}\right), \ldots, c\left(e_{5}\right)$ depending on the start vertex $v_{s}$.

\begin{tabular}{llllll}
\hline$v_{s}$ & $c\left(e_{1}\right)$ & $c\left(e_{2}\right)$ & $c\left(e_{3}\right)$ & $c\left(e_{4}\right)$ & $c\left(e_{5}\right)$ \\
\hline$v_{1}$ & $c_{1}$ & $c_{2}-c_{1}$ & 0 & $c_{3}-c_{2}$ & 0 \\
$v_{2}$ & - & $c_{2}$ & 0 & $c_{3}-c_{2}$ & 0 \\
$v_{3}$ & - & - & $c_{1}$ & $c_{2}-c_{1}$ & $c_{3}-c_{2}$ \\
$v_{4}$ & - & - & - & $c_{2}$ & $c_{3}-c_{2}$ \\
$v_{5}$ & - & - & - & - & $c_{2}$ \\
\hline
\end{tabular}

depend on the location of $v_{a}$ and $v_{b}$ in the area of zones and they also depend on a possible change at $v_{a}$. At first, let us consider the case when $v_{a}$ and $v_{b}$ are located in the same zone. If we change at $v_{a}$ then $c\left(e_{j}\right)=c_{1}$ or $c\left(e_{j}\right)=0$ otherwise, because we do not cross a zone border while running from $v_{a}$ to $v_{b}$ and it does not increase the cost of travel. In the second case, $v_{a}$ and $v_{b}$ are located in different zones. The weight equals $c\left(e_{j}\right)=c_{2}$ if we change at $v_{a}$, otherwise the value $c\left(e_{j}\right)$ depends on the number of zone borders that have been crossed since the last change while travelling to $v_{a}$. If we did not cross any zone border, then $c\left(e_{j}\right)=c_{2}-c_{1}$. It equals $c\left(e_{j}\right)=c_{3}-c_{2}$ if we have crossed a single zone border and if we have crossed two or more zone borders it equals $c\left(e_{j}\right)=0$. So, we proved that weight $c\left(e_{j}\right)$ is variable.

A determination of the value of weight $c\left(e_{j}\right)$ we illustrate by an example of running to the final vertex $v_{e}=v_{6}$ using the bus of line whose the route is shown in Fig. 2. The values $c\left(e_{1}\right), \ldots, c\left(e_{5}\right)$ depend on the start vertex $v_{s}$ from which we start the travel and they are presented in Table 1.

The bus route from the start stop $s_{s}$ to the final stop $s_{e}$ is represented by the path $p_{v_{s}, v_{e}}$ (4) from the start vertex $v_{s}$ to the final vertex $v_{e}$ in the graph $G$. In addition, the times of departures $T_{0}, T_{1}, \ldots, T_{k-1}$, where $T_{j}$ is a time of departure from $v_{j}(j=0, \ldots, k-1$; $v_{j} \in p_{v_{s}, v_{e}}$ ), are stored. Thus a path expression with reference to the graph $G$ determines the bus route in the bus network. The final solution is called the path $p_{v_{s}, v_{e}}$ and the path $p_{v_{s}, v_{i}}\left(v_{i} \neq v_{e}\right)$ is called the partial solution. The path $p_{v_{i}, v_{j}}^{\prime}=s u b_{p_{v_{s}, v_{e}}}\left(v_{i}, v_{j}\right)$ is a subpath of $p_{v_{s}, v_{e}}$ and it contains a subsequence of vertices and arcs from $v_{i}$ to $v_{j}$ belonging to $p_{v_{s}, v_{e}}$.

$$
p_{v_{s}, v_{e}}=\left\langle v_{0}=v_{s}, e_{1}, \ldots, v_{k-1}, e_{k}, v_{k}=v_{e}\right\rangle
$$

The length of the path $p_{v_{s}, v_{e}}$ is denoted by $D\left(p_{v_{s}, v_{e}}\right)$ and it equals the number of vertices belonging to the path. The path $p_{v_{s}, v_{e}}$ described by (4) has two weights $T$ and $C$, defined by (5) and (6) and its length equals $D\left(p_{v_{s}, v_{e}}\right)=k+1$. These weights represent the time and the cost of travel from $v_{s}$ to $v_{e}$ and they are equal to the sum of corresponding weights of arcs belonging to the path $p_{v_{s}, v_{e}}$. According to the formulation of the BRP 
presented in Section 2.1, the goal of the BRP is to find the path from $v_{s}$ to $v_{e}$ in the graph $G$ minimizing (5), (6) and $D\left(p_{v_{s}, v_{e}}\right)$ simultaneously.

$$
\begin{aligned}
& T\left(p_{v_{s}, v_{e}}\right)=\sum_{j=1}^{k} t\left(e_{j}\right), \\
& C\left(p_{v_{s}, v_{e}}\right)=\sum_{j=1}^{k} c\left(e_{j}\right) .
\end{aligned}
$$

The BRP is one of the problems in MOP, where we have given $k(k>1)$ criterion functions $f_{i}(i=1, \ldots, k)$ which can be minimized or maximized. In the first case the objective is to find a solution for which the value of the function is minimal and in the second case the function of determined solution should takes the maximum value. In most cases there is no a single solution for which all the criterion functions take an optimum value, i.e. the minimum or the maximum. For that reason the solution of the MOP is a set of solutions called the set of non-dominated (Pareto optimal) solutions (Coello Coello et al., 2002; Ehrgott, 1999; Ehrgott and Gandibleux, 2000; Korhonen, 1992; Pareto, 1896; Roy and Vincke, 1981; Ulungu and Teghem, 1994; Voorneveld, 2003).

Definition 1. Let there be given $k(k<1)$ minimized criterion functions $f_{1}, \ldots, f_{k}$ and two solutions $X$ and $Y$. The $X$ solution is said to dominate the $Y$ solution if $\forall i \in$ $\{1, \ldots, k\}: f_{i}(X) \leqslant f_{i}(Y)$ and $\exists j \in\{1, \ldots, k\}: f_{j}(X)<f_{j}(Y)$.

The BRP solving is reduced to solving the MSP problem between the start and the final vertices in a graph with variable weights. The solution of the problem consists of set of paths in the graph $G$ formed the set of non-dominated solutions. The weights defined by (5) and (6) and the length of the path are the criterion functions and all are minimized.

Let us analyse properties of paths belonging to the set of non-dominated solutions. The set of non-dominated solutions can contain many paths with the same values of the weights (5) and (6) and the same length, according to Definition 1 these paths are nondominated solutions. Let there be given two paths $p_{v_{s}, v_{e}}^{\prime}$ and $p_{v_{s}, v_{e}}^{\prime \prime}$, where (7) and (8) are satisfied. The paths $p_{v_{s}, v_{e}}^{\prime}$ and $p_{v_{s}, v_{e}}^{\prime \prime}$ have one of the following properties:

- $p_{v_{s}, v_{e}}^{\prime}$ and $p_{v_{s}, v_{e}}^{\prime \prime}$ differ from each other in vertices and arcs belonging to these paths,

- $p_{v_{s}, v_{e}}^{\prime}$ and $p_{v_{s}, v_{e}}^{\prime \prime}$ contain the same sequence of vertices but they differ from each other in arcs belonging to these paths,

- $p_{v_{s}, v_{e}}^{\prime}$ and $p_{v_{s}, v_{e}}^{\prime \prime}$ contain the same sequence of vertices and arcs, differ from each other only in the times of departure from all vertices belonging to these paths.

$$
\begin{aligned}
& T\left(p_{v_{s}, v_{e}}^{\prime}\right)=T\left(p_{v_{s}, v_{e}}^{\prime \prime}\right), \\
& C\left(p_{v_{s}, v_{e}}^{\prime}\right)=C\left(p_{v_{s}, v_{e}}^{\prime \prime}\right) .
\end{aligned}
$$

Let us consider the path $p_{v_{s}, v_{e}}^{\prime}$ containing a cycle $p_{v_{i}, v_{i}}=s u b_{p_{v_{s}, v_{e}}^{\prime}}\left(v_{i}, v_{i}\right)$, where $v_{i}$ is the start and the final vertex of the cycle (Fig. 3a). If there exists the path $p_{v_{s}, v_{e}}^{\prime}$, then 


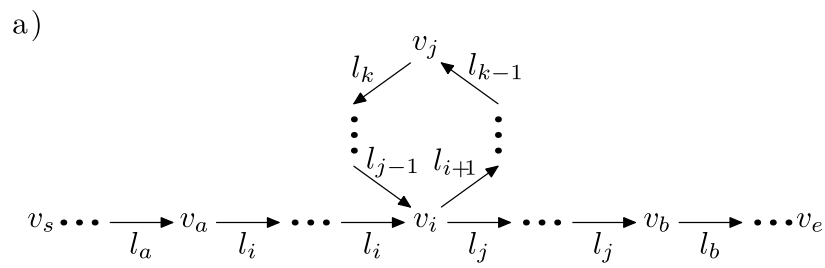

b)

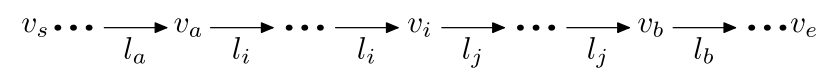

c)

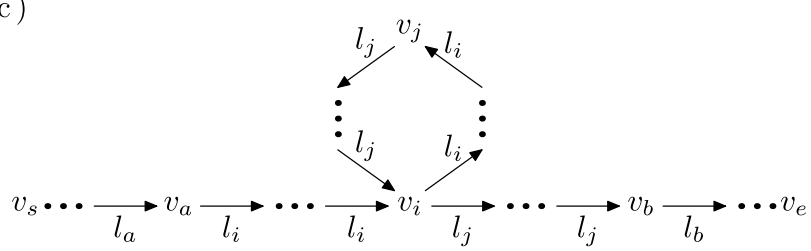

Fig. 3. A part of sample paths from the start vertex $v_{s}$ to the final vertex $v_{e}$. The arcs are described by the bus lines which the buses run between vertices.

there exists the path $p_{v_{s}, v_{e}}^{\prime \prime}$ (Fig. 3b) which has the same sequence of vertices and arcs like $p_{v_{s}, v_{e}}^{\prime}$ and it is devoid of the cycle $p_{v_{i}, v_{i}}$, i.e.:

$$
\begin{aligned}
& \operatorname{sub}_{p_{v_{s}, v_{e}}^{\prime}}\left(v_{s}, v_{i}\right)=\operatorname{sub}_{p_{v_{s}, v_{e}}^{\prime \prime}}\left(v_{s}, v_{i}\right) \\
& \operatorname{sub}_{p_{v_{s}, v_{e}}^{\prime}}\left(v_{i}, v_{e}\right)=\operatorname{sub}_{p_{v_{s}, v_{e}}^{\prime \prime}}\left(v_{i}, v_{e}\right)
\end{aligned}
$$

It has been shown that $T\left(p_{v_{s}, v_{e}}^{\prime}\right)>T\left(p_{v_{s}, v_{e}}^{\prime \prime}\right)$ and $C\left(p_{v_{s}, v_{e}}^{\prime}\right)>C\left(p_{v_{s}, v_{e}}^{\prime \prime}\right)$ if weights of arcs are constant and non-negative, and at least one is positive (Henig, 1985; Tung and Chew, 1988; Tung and Chew, 1992). In this case the set of non-dominated solutions contains only loopless paths. The graph $G$ representing the bus network has variable weights and for this case, it can be formulated Lemma 1.

Lemma 1. Let there be given the path $p_{v_{s}, v_{e}}^{\prime}$ containing the cycle $p_{v_{i}, v_{i}}$ and the path $p_{v_{s}, v_{e}}^{\prime \prime}$ which has the same sequence of vertices and arcs like $p_{v_{s}, v_{e}}^{\prime}$ and which is loopless. If weights of arcs are variable and take non-negative values then $T\left(p_{v_{s}, v_{e}}^{\prime}\right) \geqslant T\left(p_{v_{s}, v_{e}}^{\prime \prime}\right)$ and $C\left(p_{v_{s}, v_{e}}^{\prime}\right) \geqslant C\left(p_{v_{s}, v_{e}}^{\prime \prime}\right)$.

Proof. A property of the route of the bus line indicates that we change at $v_{i}$ in $p_{v_{s}, v_{e}}^{\prime \prime}$. The weights $c$ and $t$ of arcs do not take negative values and it follows that: $T\left(p_{v_{s}, v_{e}}^{\prime}\right) \geqslant$ $T\left(p_{v_{s}, v_{e}}^{\prime \prime}\right)$ and $C\left(p_{v_{s}, v_{e}}^{\prime}\right) \geqslant C\left(p_{v_{s}, v_{e}}^{\prime \prime}\right)$. The goal of the proof is to prove when conditions (7) and (8) are satisfied (both conditions are not satisfied in a graph with constant weights). The condition (9) holds, thus times of departure from $v_{s}, \ldots, v_{i-1}$ are identical in $p_{v_{s}, v_{e}}^{\prime}$ and $p_{v_{s}, v_{e}}^{\prime \prime}$ and it indicates that $T\left(s u b_{p_{v_{s}, v_{e}}^{\prime}}\left(v_{s}, v_{i}\right)\right)=T\left(s u b_{p_{v_{s}, v_{e}}^{\prime \prime}}\left(v_{s}, v_{i}\right)\right)$. If the time of waiting for a change at $v_{i}$ in $p_{v_{s}, v_{e}}^{\prime \prime}$ equals the time of making the cycle $p_{v_{i}, v_{i}}$ in $p_{v_{s}, v_{e}}^{\prime}$, 
then times of departure from vertices $v_{i}, \ldots, v_{e-1}$ in both paths are identical and (7) is satisfied. When $\delta t^{\prime \prime}=\delta t^{\prime}+T\left(p_{v_{i}, v_{i}}\right)$, where $\delta t^{\prime}$ and $\delta t^{\prime \prime}$ are the sums of times of waiting for change at the vertices in $p_{v_{s}, v_{e}}^{\prime}$ and $p_{v_{s}, v_{e}}^{\prime \prime}$, then (7) is satisfied, too.

A necessary condition for fulfilment (8) is a single change at $v_{j}$ in the cycle $p_{v_{i}, v_{i}}$ and passing $v_{i}$ without a change (Fig. 3c). For path $p_{v_{s}, v_{e}}^{\prime}$ and $p_{v_{s}, v_{e}}^{\prime \prime}$ there hold conditions:

$$
\begin{aligned}
& C\left(\operatorname{sub}_{p_{v_{s}, v_{e}}^{\prime}}\left(v_{s}, v_{a}\right)\right)=C\left(\operatorname{sub} b_{p_{v_{s}, v_{e}}^{\prime \prime}}\left(v_{s}, v_{a}\right)\right), \\
& C\left(\operatorname{sub}_{p_{v_{s}, v_{e}}^{\prime}}\left(v_{b}, v_{e}\right)\right)=C\left(\operatorname{sub} b_{p_{v_{s}, v_{e}}^{\prime \prime}}\left(v_{b}, v_{e}\right)\right) .
\end{aligned}
$$

If all vertices belonging to the cycle $p_{v_{i}, v_{i}}$ are located in the same zone, then conditions (11) and (12) are satisfied and (8) is satisfied, too.

$$
\begin{aligned}
& C\left(\operatorname{sub}_{p_{v_{s}, v_{e}}^{\prime}}\left(v_{a}, v_{j}\right)\right)=C\left(\operatorname{sub}{p_{v_{s}, v_{e}}^{\prime \prime}}\left(v_{a}, v_{i}\right)\right) \\
& C\left(\operatorname{sub}_{p_{v_{s}, v_{e}}^{\prime}}\left(v_{j}, v_{b}\right)\right)=C\left(\operatorname{sub}_{p_{v_{s}, v_{e}}^{\prime \prime}}\left(v_{i}, v_{b}\right)\right) .
\end{aligned}
$$

If the vertices belonging to the cycle $p_{v_{i}, v_{i}}$ are located in different zones, then (11) is satisfied when:

- we do not cross two or more zones when we run from $v_{a}$ to $v_{i}$ and we do not cross any zone when we run from $v_{i}$ to $v_{j}$ in $p_{v_{i}, v_{i}}$, or

- we cross two or more zones when we run from $v_{a}$ to $v_{i}$.

The condition (12) is satisfied in similar cases, i.e.:

- we do not cross any zone when we run from $v_{j}$ to $v_{i}$ in $p_{v_{i}, v_{i}}$, if we do not cross two or more zones when we run from $v_{i}$ to $v_{b}$, or

- we cross two or more zones when we run from $v_{i}$ to $v_{b}$.

To illustrate Lemma 1 let us consider a sample routes from $v_{s}=1$ to $v_{e}=4$, where the time of starting travel equals $T_{s}=8: 00$. The route $p_{1,4}^{\prime}$ without a cycle is presented in Tables 2 and 3 the route $p_{1,4}^{\prime \prime}$ containing a cycle is presented. All vertices belonging to these paths are located in the same zone and (13), (14) are satisfied. In the route $p_{1,4}^{\prime}$ we change at vertex 3 and the time of waiting for a change equals 45 minutes and it equals the time of making the cycle $3 \rightarrow 5 \rightarrow 3$ in the route $p_{1,4}^{\prime \prime}$. In addition, (15) and (16) occur and for that reason the cost of making the cycle does not increase the total cost of travel.

$$
\begin{aligned}
& T\left(p_{1,4}^{\prime}\right)=T\left(p_{1,4}^{\prime \prime}\right)=65 \text { minutes, } \\
& C\left(p_{1,4}^{\prime}\right)=C\left(p_{1,4}^{\prime \prime}\right)=6.0 \text { units, } \\
& C\left(s u b_{p_{1,4}^{\prime}}(2,3)\right)=C\left(s u b_{p_{1,4}^{\prime \prime}}(2,5)\right)=2.0 \text { units, } \\
& C\left(s u b_{p_{1,4}^{\prime}}(3,4)\right)=C\left(s u b_{p_{1,4}^{\prime \prime}}(5,4)\right)=2.0 \text { units. }
\end{aligned}
$$

The routes $p_{1,4}^{\prime}$ and $p_{1,4}^{\prime \prime}$ show that weights of arcs representing the time and the cost of travel are variable. Let us consider the arc $(3,4)$. In the route $p_{1,4}^{\prime}$ weights of this arc 
Table 2

A timetable of the route from $v_{s}=1$ to $v_{e}=4$ without a cycle, where the time of starting travel equals $T_{s}=8: 00$.

\begin{tabular}{lllll}
\hline Vertex/zone & Arrival time & Departure time & Cost of travel & Line \\
\hline $1 / 1$ & $8: 00$ & $8: 05$ & 0.0 & 1 \\
$2 / 1$ & $8: 08$ & $8: 12$ & 2.0 & 2 \\
$3 / 1$ & $8: 15$ & $9: 00$ & 4.0 & 3 \\
$4 / 1$ & $9: 05$ & & 6.0 & \\
\hline
\end{tabular}

Table 3

A timetable of the route from $v_{s}=1$ to $v_{e}=4$ containing a cycle $3 \rightarrow 5 \rightarrow 3$, where the time of starting travel equals $T_{S}=8: 00$.

\begin{tabular}{lllll}
\hline Vertex/zone & Arrival time & Departure time & Cost of travel & Line \\
\hline $1 / 1$ & $8: 00$ & $8: 05$ & 0.0 & 1 \\
$2 / 1$ & $8: 08$ & $8: 12$ & 2.0 & 2 \\
$3 / 1$ & $8: 15$ & $8: 15$ & 4.0 & 2 \\
$5 / 1$ & $8: 20$ & $8: 55$ & 4.0 & 3 \\
$3 / 1$ & $9: 00$ & $9: 00$ & 6.0 & 3 \\
$4 / 1$ & $9: 05$ & & 6.0 & \\
\hline
\end{tabular}

equal $t(3,4)=50$ minutes and $c(3,4)=2.0$ units, but in the route $p_{1,4}^{\prime \prime}$ these weights equal $t(3,4)=5$ minutes and $c(3,4)=0.0$ units.

It should be pointed out that the path $p_{v_{s}, v_{e}}^{\prime}$ defined by Lemma 1 for which (7) and (8) are satisfied is dominated by the path $p_{v_{s}, v_{e}}^{\prime \prime}$ due to $D\left(p_{v_{s}, v_{e}}^{\prime}\right)>D\left(p_{v_{s}, v_{e}}^{\prime \prime}\right)$. The last property of routes belonging to the set of non-dominated solutions is determined by Lemma 2.

Lemma 2. Let $p_{v_{s}, v_{e}}$ and $p_{v_{s}, v_{e}}^{\prime}$ be paths which consist of the same sequence of vertices and arcs but differ from each other in times of departure from vertices belonging to these paths. Both paths are non-dominated solutions if we change once at least in these paths.

Proof. If we do not a change in $p_{v_{s}, v_{e}}$ and $p_{v_{s}, v_{e}}^{\prime}$, it follows that departure time from $v_{s}$ are different in these paths and departure times from all vertices belonging to these paths are different, too. For that reason $T\left(p_{v_{s}, v_{e}}\right) \neq T\left(p_{v_{s}, v_{e}}^{\prime}\right)$ and one of them is a dominated solution.

Let these paths consist of sequence of vertices and arcs:

$$
p_{v_{s}, v_{e}}=p_{v_{s}, v_{e}}^{\prime}=\left\langle v_{s}, \ldots, e_{j}, v_{j}, e_{k}, \ldots, e_{k}, v_{k}, e_{i}, \ldots, v_{e}\right\rangle
$$

where $v_{j}$ and $v_{k}$ are vertices where we change. Let the arrival times at $v_{j}$ and $v_{k}$ are equal $T_{a j}, T_{a k}$, and $T_{d j}, T_{d k}$ be departure times from $v_{j}$ and $v_{k}$, and the time of waiting for a change at $v_{k}$ equals $\delta t_{k}=T_{d k}-T_{a k}$. If it is possible to leave $v_{j}$ at time $T_{d j}^{\prime}>T_{d j}$ in $p_{v_{s}, v_{e}}^{\prime}$, where $T_{d j}^{\prime}-T_{d j} \leqslant \delta t_{k}$, then it is possible to leave $v_{k}$ at time $T_{d k}$, and it does not increase the total time of travel from $v_{s}$ to $v_{e}$ in the path $p_{v_{s}, v_{e}}^{\prime}$, i.e., $T\left(p_{v_{s}, v_{e}}\right)=T\left(p_{v_{s}, v_{e}}^{\prime}\right)$. 
Table 4

Timetables of the routes from $v_{s}=6$ to $v_{e}=10$, where the time of starting travel equals $T_{s}=8: 00$.

\begin{tabular}{clllllll}
\hline Vertex & \multicolumn{3}{l}{ Arrival times } & \multicolumn{5}{c}{ Departure times } & Line \\
\hline 6 & $8: 00$ & $8: 00$ & $8: 00$ & $8: 10$ & $8: 25$ & $8: 40$ & 4 \\
7 & $8: 15$ & $8: 30$ & $8: 45$ & $8: 15$ & $8: 30$ & $8: 45$ & 4 \\
8 & $8: 25$ & $8: 40$ & $8: 55$ & $9: 00$ & $9: 00$ & $9: 00$ & 5 \\
9 & $9: 05$ & $9: 05$ & $9: 05$ & $9: 05$ & $9: 05$ & $9: 05$ & 5 \\
10 & $9: 10$ & $9: 10$ & $9: 10$ & & & & \\
\hline
\end{tabular}

a)

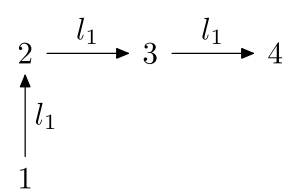

b)

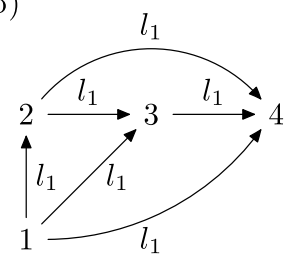

c)

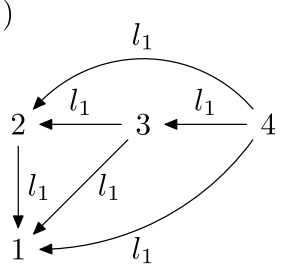

Fig. 4. The route of the bus line $l_{1}$ represented by the graphs $G, G_{a}$ and $G_{a r}$.

Lemma 2 is illustrated in Table 4 , where a sample routes from $v_{s}=6$ to $v_{e}=10$ are presented. There are three routes with the same time and cost of travel which contain the same sequence of vertices and arcs, but departure times from vertex $v_{s}=6$ are different.

\section{The Algorithm to Solving the BRP}

In this section we present an algorithm for finding all routes from the start vertex $v_{s}$ to the final vertex $v_{e}$, belonging to the set of non-dominated solutions. The algorithm belongs to a group of label correcting algorithms with storing partial solutions and current elimination of dominated solutions. The algorithm is presented in the form of the procedure FindRoutes (Fig. 5). The bus network is represented by the graph $G=(V, E)$ and additionally it is represented by other graphs: $G_{a}=\left(V, E_{a}\right), G_{a r}=\left(V, E_{a r}\right)$. We create the graph $G_{a}$ by adding into the set $E$ additional arcs for each bus line according to the following principles. Let the route of $i$-th bus line is described by (2). For each vertices $v_{j}^{i}(j=0, \ldots, k-2)$ and $v_{u}^{i}(u=j+2, \ldots, k)$ we add arc $\left(v_{j}^{i}, v_{u}^{i}\right)$. For example, let us consider the bus network represented by the graph $G$ where buses of the bus line $l_{1}$ are run (Fig. 4a). The graph $G_{a}$ (Fig. 4b) is obtained by adding arcs $(1,3),(1,4)$ and $(2,4)$ into the set $E$. The graph $G_{a r}$ is created by reversing all arcs of $E_{a}$ to the opposite direction (Fig. 4c).

During the process of finding the solutions the following data structures are used: $S$, $P S$ and $Q$. The $S$ structure is a list of final solutions formed as the set of non-dominated solutions. Each solution represents the path from $v_{s}$ to $v_{e}$, where the time of starting travel 
Input: $\quad G, G_{a}, G_{a r}-$ graphs representing the bus network

$v_{s}, v_{e} \quad-$ vertices representing the start and the final stops

$T_{s} \quad-\quad$ the time of starting travel at the start stop

Output: $S \quad-$ list of computed routes

procedure FindRoutes $\left(G, G_{a}, G_{a r}, v_{s}, v_{e}, T_{s}, S\right)$

$P_{t} \leftarrow \operatorname{PathMinT}\left(G_{a}, v_{s}, v_{e}, T_{s}\right) ; \quad \bar{t}_{m}^{e} \leftarrow \operatorname{MinT}\left(G_{a r}, v_{e}\right) ;$

$P_{c} \leftarrow \operatorname{PathMinC}\left(G_{a}, v_{s}, v_{e}, T_{s}\right) ; \quad \bar{c}_{m}^{e} \leftarrow \operatorname{MinC}\left(G_{a r}, v_{e}\right) ;$

$P_{d} \leftarrow \operatorname{PAThMinD}\left(G_{a}, v_{s}, v_{e}, T_{s}\right) ; \quad \bar{d}_{m}^{e} \leftarrow \operatorname{MinD}\left(G_{a r}, v_{e}\right) ;$

for all $v_{i} \in V$ do

$P S\left[v_{i}\right] \Leftarrow \emptyset$

end for

$P S\left[v_{s}\right] \Leftarrow\left(0,0,0,1,\left(0, T_{s}\right.\right.$, null $\left.)\right) ; \quad \operatorname{PUSH}\left(Q, v_{s}\right) ;$

while $Q \neq \emptyset$ do

$v_{i} \leftarrow \operatorname{POP}(Q) ;$

for all $\left(v_{i}, v_{j}, l_{j}\right) \in \operatorname{out}\left(v_{i}\right) ; v_{j} \neq v_{s}$ do

for all $P \in \operatorname{not}$ analysed solutions in $P S\left[v_{i}\right]$ do

if not $\operatorname{BelongsToPath}\left(P, v_{j}\right)$ and $P . l_{j} \neq l_{j}$ then

$T_{i} \leftarrow$ time of departure from $v_{i}$

$d_{s j} \leftarrow$ length of the route from $v_{s}$ to $v_{j}$;

$t_{s j}, c_{s j} \leftarrow$ the time and the cost of travel from $v_{s}$ to $v_{j}$;

if $\operatorname{NotDom}\left(t_{s j}, c_{s j}, d_{s j}, P_{t}, P_{c}, P_{d}, \bar{t}_{m}^{e}, \bar{c}_{m}^{e}, \bar{d}_{m}^{e}\right)$ then

$P_{j} \leftarrow$ CheckSolution $\left(t_{s j}, c_{s j}, l_{j}, d_{s j}, P S\left[v_{j}\right]\right) ;$

if $P_{j} \neq$ null then $\quad$ exists solution $P_{j}$ in the list $\left.P S\left[v_{j}\right]\right\}$

$\operatorname{Push}\left(P_{j} . L P,\left(v_{i}, T_{i}, \& P\right)\right)$;

else

$P^{\prime} \leftarrow\left(l_{j}, t_{s j}, c_{s j}, d_{s j},\left(v_{i}, T_{i}, \& P\right)\right)$

if $v_{j}=v_{e}$ then

else $P S\left[v_{j}\right] \Leftarrow \operatorname{AdDToFinalList}\left(P S\left[v_{j}\right], P^{\prime}\right) ;$

end if

$P S\left[v_{j}\right] \Leftarrow \operatorname{AdDToList}\left(P S\left[v_{j}\right], P^{\prime}\right) ; \quad \operatorname{Push}\left(Q, v_{j}\right) ;$

end if

end if

end if

end for

end for

end while

$S \Leftarrow \operatorname{CreateFullPaths}\left(P S\left[v_{e}\right], v_{e}\right) ; \quad S \Leftarrow \operatorname{PathidifferTimes}(S, G)$;

: end procedure

Fig. 5. The algorithm for finding all routes from the start vertex $v_{s}$ to the final vertex $v_{e}$ belonging to the set of non-dominated solutions.

at $v_{s}$ equals $T_{s}$. For each vertex $v_{j} \in V$ the $P S\left[v_{j}\right]$ structure contains a list of partial solutions from $v_{s}$ to $v_{j}$ and for $v_{e}$ it contains a list of final solutions and it constitute the set of non-dominated solutions. The partial and the final solutions are described by a record $\left(l_{j}, t_{s j}, c_{s j}, d_{s j}, L P\right)$, where: 
a)

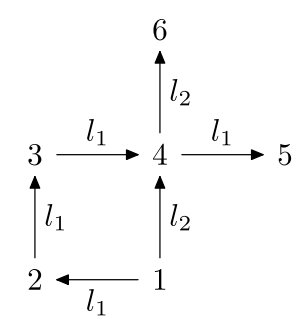

b)

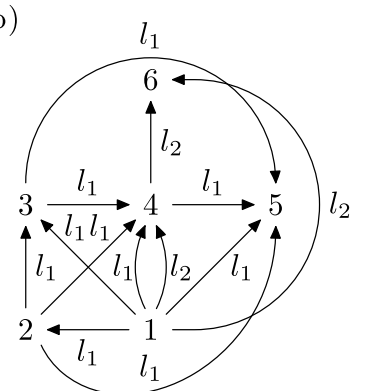

Fig. 6. A sample bus network represented by the graphs $G$ and $G_{a}$.

- $l_{j}$ - the bus line by which we travel to $v_{j}$ from a vertex which precedes $v_{j}$ in the path,

- $t_{s j}, c_{s j}$ - the time and the cost of travel from $v_{s}$ to $v_{j}$ (for $v_{s}$ they equal 0 ),

- $d_{s j}$ - the length of the path from $v_{s}$ to $v_{j}$,

- $L P$ - a list of records $\left(v_{i}, T_{i}, * P_{i}\right)$, where:

- $v_{i}$ - the vertex which precedes the vertex $v_{j}$ in the path,

- $T_{i}$ - time of departure from $v_{i}$ towards $v_{j}$ (for $v_{s}$ it equals the time of starting travel $T_{S}$ ),

- $* P_{i}-$ a pointer to the partial solution for the vertex $v_{i}$.

The $Q$ structure is a queue containing vertices for which the partial solutions have been determined.

The initial part of the algorithm (lines 2-8) contains finding the paths of the minimal time and the minimal cost of travel and the minimal length from $v_{s}$ to $v_{e}$ in the graph $G_{a}$. These paths are stored in $P_{t}, P_{c}$ and $P_{d}$, and they are computed by the PathMinT (line 2), the PathMinC (line 3) and the PathMinD procedures (line 4), where the first two procedures implement the Dijkstra algorithm and the last implements Breadth-First-Search method (Jungnickel, 1989). We can prove that it is not possible to find the path of the minimal cost of travel in the graph $G$ using shortest paths algorithms like the Dijkstra or the Bellman-Ford. Let us consider the graph $G$ representing the bus network where we want to determine the path of the minimal cost of travel from $v_{s}=1$ to $v_{e}=5$ (Fig. 6a). If we assume that all vertices belong to the same zone except for vertices 2 and 3 , then $1 \rightarrow 4 \rightarrow 5$ is the path of the minimal cost of travel determined in the graph $G$ by the Dijkstra or the Bellman-Ford algorithm and it has cost 4.0 units. This path is incorrect because the path of minimal cost of travel has form $1 \rightarrow 2 \rightarrow 3 \rightarrow 4 \rightarrow 5$ and its cost equals 2.6 units. This path is determined by the Dijkstra or the Bellman-Ford algorithm in the graph $G_{a}$.

The Dijkstra algorithm is also used in the procedures MINT (line 2) and MinC (line 3) for determining minimal costs and times of travel from all vertices to the final vertex $v_{e}$. Determined values are stored in arrays $\bar{c}_{m}^{e}$ and $\bar{t}_{m}^{e}$, where $\bar{c}_{m}^{e}\left[v_{j}\right]$ and $\bar{t}_{m}^{e}\left[v_{j}\right]$ stores adequately the minimal cost and time of travel from $v_{j}$ to $v_{e}$. The last array $\bar{d}_{m}^{e}\left[v_{j}\right]$ stores minimal lengths of paths from all vertices to the final vertex $v_{e}$. It is determined by the 


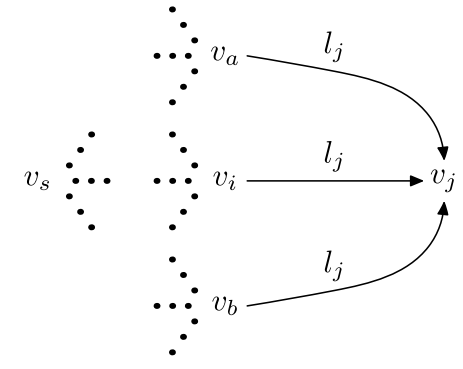

Fig. 7. The vertices which precede the vertex $v_{j}$ in the route.

procedure MinD (line 4), which implements Breadth-First-Search method. For each vertex $v_{i} \neq v_{s}$ the $P S\left[v_{i}\right]$ structure is initialised by the empty list (lines 5-7). The $P S\left[v_{s}\right]$ structure of the start vertex $v_{s}$ is initialised by a record representing an initial solution from which the algorithm starts determining all solutions and $v_{s}$ is inserted into the queue (line 8).

In the while-loop (lines 9-33) all non-dominated paths are computed by visiting the vertices of the graph $G_{a}$ using a modified Breadth-First-Search method. We do not compute paths which differ from each other only in times of departure from vertices, because these paths are computed (line 34) based on the paths computed in the while-loop.

A single iteration contains following operations. The first vertex $v_{i}$ is taken from the queue $Q$ (line 10) and all its outgoing arcs $\left(v_{i}, v_{j}\right)$ are analysed (lines 11-32). For arc $\left(v_{i}, v_{j}\right)$ we try to extend each the solution $P$ belonging the list $P S\left[v_{i}\right]$ and create a new solution for the vertex $v_{j}$ (lines 12-31). The new solution represents a path from $v_{s}$ to $v_{j}$ where $v_{j}$ is preceded by $v_{i}$ in the path. During the process of finding the solutions the graph $G_{a}$ containing additional arcs is used and it allows to examine all reachable vertices from $v_{i}$ by the bus of the bus line $l_{j}$. For that reason only vertices where we change are added to the path. Thus, we extend the solution $P$ if all conditions are satisfied:

- the solution $P$ has not been analysed yet (checking in the line 12),

- the vertex $v_{j}$ does not belong to the path represented by the solution $P$ (it is checked by the procedure BelongsToPath in the line 13),

- the bus is changed at $v_{i}$ (checking in the line 13).

If all mentioned conditions are satisfied, the time of departure from $v_{i}$, the length of the route, the time and the cost of travel from $v_{s}$ to $v_{j}$ are determined for the extended solution (lines 14-16). In the next, the procedure NotDom is used to determine if it is possible to obtain a non-dominated final solution from the extended solution (line 17). When the estimation is positive, we try to add the extended solution to the list $P S\left[v_{j}\right]$.

The vertex $v_{j}$ can be reached from many vertices by the bus of line $l_{j}$ (Fig. 7). In the Fig. 7, there are three ways to travel to $v_{j}$ and each of the cases represents a different path from $v_{s}$ to $v_{j}$. For that reason, there may exist many paths with the same length, time and cost of travel where we run to $v_{j}$ by bus of the same bus line. In this case the list $P S\left[v_{j}\right]$ contains only a single record which represents all these paths. It reduces the number of 
stored and analysed solutions. Thus, before creating a new record representing the path we check using the procedure CHeckSolution if the record $P_{j}$, where the time and the cost of travel are equal $t_{s j}$ and $c_{s j}$, the length of the route equals $d_{s j}$ and where we run to $v_{j}$ by bus of the line $l_{j}$, exists in the list $P S\left[v_{j}\right]$ (line 18). If $P_{j}$ exists we only add a record $\left(v_{i}, T_{i}, \& P\right)$ to the list $P_{j} \cdot L P$ stored in $P_{j}$ (line 20). Otherwise, a new record $P^{\prime}$ is created (line 22) and it is added to $P S\left[v_{j}\right]$ (lines 24, 26). If $P^{\prime}$ is created for the final vertex $v_{e}$, there is used the procedure AdDToFinalList (line 24) which add $P^{\prime}$ to the list $P S\left[v_{j}\right]$ and removes dominated solutions. Otherwise, there is used the procedure ADDToList and $v_{j}$ is added to the queue $Q$ (line 26). We finish the computation in the while-loop when the queue $Q$ is empty and it means that we have searched a space of solutions.

In the last part of the algorithm we use the procedure CreateFullPaths to determine full routes for all computed solutions represented by records stored in the list $P S\left[v_{e}\right]$ (line 34). The full route is determined based on the values stored in the record, i.e. the vertex $v_{i}$ which precedes the vertex $v_{e}$ in the path and the pointer $* P_{i}$ to the partial solution for $v_{i}$. In the last step of the algorithm the paths, that differ from each other only in times of departure from all vertices belonging to the path, are determined using the procedure PATHsDifferTimes. In the procedure all paths belonging to the $S$ list are analysed (line 34). Let us consider the path $P \in S$ containing a sequence vertices of changes: $v_{0}=v_{s}, v_{1}, \ldots, v_{k-1}, v_{k}=v_{e}$, where times of departure from these vertices are equal respectively: $T_{0}, T_{1}, \ldots, T_{k-1}$. A new solution $P^{\prime}$ is created and added to the list $S$ if one can leave $v_{i}(0 \leqslant i<k-1)$ at any time later than $T_{i}$ and arrive to $v_{e}$ at the same time as in $P$. The solution $P^{\prime}$ differs from $P$ only in times of departure from vertices belonging to the path, and as a consequence differs in times of waiting for changes, but total times of waiting in both paths are equal.

The extended solution represents a path from $v_{s}$ to $v_{j}$, its length equals $d_{s j}$, the time and the cost of travel equal $t_{s j}$ and $c_{s j}$ and it is estimated if it is possible to obtain a nondominated final solution from it. The estimation is made on the basis of determined paths $P_{t}, P_{c}, P_{d}$ and values $\bar{c}_{m}^{e}\left[v_{j}\right], \bar{t}_{m}^{e}\left[v_{j}\right], \bar{d}_{m}^{e}\left[v_{j}\right]$ using the procedure NoтDom (line 17). Let $t_{\max }$ equals the maximal time, $c_{\max }$ equals the maximal cost of travel and $d_{\max }$ equals the maximal length from among paths $P_{t}, P_{c}$ and $P_{d}$, what is described by (17)-(19).

$$
\begin{aligned}
& t_{\max }=\max \left\{T\left(P_{t}\right), T\left(P_{c}\right), T\left(P_{d}\right)\right\}, \\
& c_{\max }=\max \left\{C\left(P_{t}\right), C\left(P_{c}\right), C\left(P_{d}\right)\right\}, \\
& d_{\max }=\max \left\{D\left(P_{t}\right), D\left(P_{c}\right), D\left(P_{d}\right)\right\} .
\end{aligned}
$$

The minimal time of travel from $v_{j}$ to $v_{e}$ equals $\bar{t}_{m}^{e}\left[v_{j}\right]$, for that reason the value of the time of travel $t_{s e}$ the final solution obtained from the extended solution is defined by (20). In the same way are defined the cost of travel $c_{s e}$ and the length of the path $d_{s e}(21),(22)$.

$$
\begin{aligned}
& t_{s e} \geqslant t_{s j}+\bar{t}_{m}^{e}\left[v_{j}\right], \\
& c_{s e} \geqslant c_{s j}+\bar{c}_{m}^{e}\left[v_{j}\right], \\
& d_{s e} \geqslant d_{s j}+\bar{d}_{m}^{e}\left[v_{j}\right] .
\end{aligned}
$$


It is not possible to obtain a non-dominated final solution from the extended solution if one of conditions (23)-(25) is satisfied. These conditions are checked in the procedure NotDom. Additionally, there is checked if paths $P_{t}, P_{c}, P_{d}$ dominate the final solution obtained from the extended solution. It is checked by comparing values $t_{s j}+\bar{t}_{m}^{e}\left[v_{j}\right], c_{s j}+$ $\bar{c}_{m}^{e}\left[v_{j}\right]$ and $d_{s j}+\bar{d}_{m}^{e}\left[v_{j}\right]$ with the time and the cost of travel and the length of paths $P_{t}$, $P_{c}, P_{d}$ using Definition 1 .

$$
\begin{aligned}
& t_{s j}+\bar{t}_{m}^{e}\left[v_{j}\right]>t_{\max } \wedge c_{s j}+\bar{c}_{m}^{e}\left[v_{j}\right] \geqslant c_{\max } \wedge d_{s j}+\bar{d}_{m}^{e}\left[v_{j}\right] \geqslant d_{\max }, \\
& t_{s j}+\bar{t}_{m}^{e}\left[v_{j}\right] \geqslant t_{\max } \wedge c_{s j}+\bar{c}_{m}^{e}\left[v_{j}\right]>c_{\max } \wedge d_{s j}+\bar{d}_{m}^{e}\left[v_{j}\right] \geqslant d_{\max }, \\
& t_{s j}+\bar{t}_{m}^{e}\left[v_{j}\right] \geqslant t_{\max } \wedge c_{s j}+\bar{c}_{m}^{e}\left[v_{j}\right] \geqslant c_{\max } \wedge d_{s j}+\bar{d}_{m}^{e}\left[v_{j}\right]>d_{\max } .
\end{aligned}
$$

The procedure AdDToList adds the record $P^{\prime}$ representing the path $p_{v_{s}, v_{j}}^{\prime}$ to the list $P S\left[v_{j}\right]$ (line 26) and estimates it based on already existing records. It is possible to determine whether the final solution $p_{v_{s}, v_{e}}^{\prime}$ obtained from $P^{\prime}$ is dominated. Let $P^{\prime \prime}$ is already existing record belonging to $P S\left[v_{j}\right]$ and it represents the path $p_{v_{s}, v_{j}}^{\prime \prime}$. If (26) is satisfied, it is possible to obtain the final solution $p_{v_{s}, v_{e}}^{\prime \prime}$ from $p_{v_{s}, v_{j}}^{\prime \prime}$, where the time of departure from $v_{j}$ is the same as in $p_{v_{s}, v_{e}}^{\prime}$ and it follows that the time of travel meets (27).

$$
\begin{aligned}
& T\left(p_{v_{s}, v_{j}}^{\prime}\right)=T\left(p_{v_{s}, v_{j}}^{\prime \prime}\right), \\
& T\left(p_{v_{s}, v_{e}}^{\prime}\right)=T\left(p_{v_{s}, v_{e}}^{\prime \prime}\right) .
\end{aligned}
$$

The relationships between the lengths of the paths $p_{v_{s}, v_{j}}^{\prime}$ and $p_{v_{s}, v_{j}}^{\prime \prime}$ and the lengths of obtained the final solutions $p_{v_{s}, v_{e}}^{\prime}$ and $p_{v_{s}, v_{e}}^{\prime \prime}$ are described by (28)-(30).

$$
\begin{aligned}
& D\left(p_{v_{s}, v_{j}}^{\prime}\right)>D\left(p_{v_{s}, v_{j}}^{\prime \prime}\right) \quad \Rightarrow \quad D\left(p_{v_{s}, v_{e}}^{\prime}\right)>D\left(p_{v_{s}, v_{e}}^{\prime \prime}\right) \\
& D\left(p_{v_{s}, v_{j}}^{\prime}\right)=D\left(p_{v_{s}, v_{j}}^{\prime \prime}\right) \quad \Rightarrow \quad D\left(p_{v_{s}, v_{e}}^{\prime}\right)=D\left(p_{v_{s}, v_{e}}^{\prime \prime}\right) \\
& D\left(p_{v_{s}, v_{j}}^{\prime}\right)<D\left(p_{v_{s}, v_{j}}^{\prime \prime}\right) \quad \Rightarrow \quad D\left(p_{v_{s}, v_{e}}^{\prime}\right)<D\left(p_{v_{s}, v_{e}}^{\prime \prime}\right) .
\end{aligned}
$$

If the costs of travel meet (31) then (32) is satisfied and if (28) or (29) is satisfied then $P^{\prime}$ is not added to the list $P S\left[v_{j}\right]$ because the final solution $p_{v_{s}, v_{e}}^{\prime}$ obtained from $P^{\prime}$ is dominated by $p_{v_{s}, v_{e}}^{\prime \prime}$. Otherwise, i.e. if (33) is satisfied, it is not possible to determine the relationship between $C\left(p_{v_{s}, v_{e}}^{\prime}\right)$ and $C\left(p_{v_{s}, v_{e}}^{\prime \prime}\right)$ and $P^{\prime}$ is added to $P S\left[v_{j}\right]$.

$$
\begin{aligned}
& C\left(p_{v_{s}, v_{j}}^{\prime}\right)>C\left(p_{v_{s}, v_{j}}^{\prime \prime}\right), \\
& C\left(p_{v_{s}, v_{e}}^{\prime}\right)>C\left(p_{v_{s}, v_{e}}^{\prime \prime}\right), \\
& C\left(p_{v_{s}, v_{j}}^{\prime}\right) \leqslant C\left(p_{v_{s}, v_{j}}^{\prime \prime}\right) .
\end{aligned}
$$

If (34) is satisfied, then (35) is also satisfied and we do not add $P^{\prime}$ to $P S\left[v_{j}\right]$ only when (28) or (29) occur and additionally (36) occurs. If (28) or (29) and (36) are satisfied then $p_{v_{s}, v_{e}}^{\prime \prime}$ dominates $p_{v_{s}, v_{e}}^{\prime}$. 


$$
\begin{aligned}
& T\left(p_{v_{s}, v_{j}}^{\prime}\right)>T\left(p_{v_{s}, v_{j}}^{\prime \prime}\right), \\
& T\left(p_{v_{s}, v_{e}}^{\prime}\right) \geqslant T\left(p_{v_{s}, v_{e}}^{\prime \prime}\right), \\
& C\left(p_{v_{s}, v_{j}}^{\prime}\right)>C\left(p_{v_{s}, v_{j}}^{\prime \prime}\right) .
\end{aligned}
$$

In the last case, (37) occurs and it follows that (38) is satisfied. In the case when it is not possible to determine the relationship between $C\left(p_{v_{s}, v_{e}}^{\prime}\right)$ and $C\left(p_{v_{s}, v_{e}}^{\prime \prime}\right)$, we have to add $P^{\prime}$ to the list $P S\left[v_{j}\right]$.

$$
\begin{aligned}
& T\left(p_{v_{s}, v_{j}}^{\prime}\right)<T\left(p_{v_{s}, v_{j}}^{\prime \prime}\right), \\
& T\left(p_{v_{s}, v_{e}}^{\prime}\right) \leqslant T\left(p_{v_{s}, v_{e}}^{\prime \prime}\right) .
\end{aligned}
$$

To sum up, the record $P^{\prime}$ is not added to the list $P S\left[v_{j}\right]$ when (39) are satisfied and these conditions are used in the procedure ADDToList.

$$
T\left(p_{v_{s}, v_{j}}^{\prime}\right) \geqslant T\left(p_{v_{s}, v_{j}}^{\prime \prime}\right) \wedge C\left(p_{v_{s}, v_{j}}^{\prime}\right)>C\left(p_{v_{s}, v_{j}}^{\prime \prime}\right) \wedge D\left(p_{v_{s}, v_{j}}^{\prime}\right) \geqslant D\left(p_{v_{s}, v_{j}}^{\prime \prime}\right) .
$$

If (40) and (41) are satisfied, then $P^{\prime}$ is dominated by $P^{\prime \prime}$ but it is added to the list $P S\left[v_{j}\right]$. Thus, the list $P S\left[v_{j}\right]$ of the vertex $v_{j} \neq v_{e}$ can contains records being dominated solutions and a non-dominated final solution for the final vertex $v_{e}$ can be obtained from a dominated partial solution for $v_{j} \neq v_{e}$. This is an important property of a graph with variable weight and it is not satisfied in a graph with constant weight. It has been shown, that it is not possibly to obtain a non-dominated final solution from a dominated partial solution if weights are constant (Azevedo and Martins, 1991; Martins et al., 1999; Mote et al., 1991).

$$
\begin{aligned}
& T\left(p_{v_{s}, v_{j}}^{\prime}\right)>T\left(p_{v_{s}, v_{j}}^{\prime \prime}\right) \wedge C\left(p_{v_{s}, v_{j}}^{\prime}\right)=C\left(p_{v_{s}, v_{j}}^{\prime \prime}\right) \wedge D\left(p_{v_{s}, v_{j}}^{\prime}\right) \geqslant D\left(p_{v_{s}, v_{j}}^{\prime \prime}\right) \\
& T\left(p_{v_{s}, v_{j}}^{\prime}\right) \geqslant T\left(p_{v_{s}, v_{j}}^{\prime \prime}\right) \wedge C\left(p_{v_{s}, v_{j}}^{\prime}\right)=C\left(p_{v_{s}, v_{j}}^{\prime \prime}\right) \wedge D\left(p_{v_{s}, v_{j}}^{\prime}\right)>D\left(p_{v_{s}, v_{j}}^{\prime \prime}\right) .
\end{aligned}
$$

A time complexity of the algorithm FINDRoutes depends on a number of paths belonging to the set of non-dominated solutions. The MSP problem is known to be NP-complete and it has been shown that the number of non-dominated solutions is exponential at least in the worst case which implies that any deterministic algorithm that attempts to solve it is also exponential in terms of pessimistic time complexity. The maximum number of nondominated solutions equals $g^{n-1}$, where $n$ is a number of vertices and $g$ is a maximum out-degree of a vertex in the graph (Skriver and Andersen, 2000b). A time complexity of the algorithm is clearly dominated by execution of the while-loop (lines 9-33). Each of computed paths can contain at most $n$ vertices. In the line 10 the vertex $v_{i}$ is taken from the queue $Q$ and $g_{i}$ arcs are examined, where $g_{i}$ is the out-degree of the vertex $v_{i}$. Thus, in the worst case it is necessary to examine $\prod_{i=1}^{n-1} d_{i}$ arcs and the pessimistic time complexity equals $O\left(d^{n}\right)$, where $g=\max _{i=1, \ldots, n-1}\left\{d_{i}\right\}$. 
Table 5

Parameters of the graphs representing the bus network.

\begin{tabular}{lrc}
\hline & $G$ & $G_{a}, G_{a r}$ \\
\hline Number of vertices & 1211 & 1211 \\
Number of arcs & 5549 & 35610 \\
Max. in-degree of vertex & 13 & 122 \\
Max. out-degree of vertex & 13 & 122 \\
Min. in-degree of vertex & 1 & 6 \\
Min. out-degree of vertex & 1 & 6 \\
Max. degree of vertex & 26 & 244 \\
Min. degree of vertex & 2 & 13 \\
\hline
\end{tabular}

\section{Experimental results}

The procedure FindRoutes (Fig. 5) has been implemented in C++ and the test experiments were carried out on $2.66 \mathrm{GHz}$ Pentium-IV computer with 1 GB of RAM, running under Windows Server 2003 Enterprise Edition. In addition we have adapted the Brumbaugh-Smith (Brumbaugh-Smith and Shier, 1989) and the Skriver (Skriver and Andersen, 2000b) algorithms to variable weights and both algorithms have been implemented in $\mathrm{C}++$. In the tests we used a bus network consisting of 1211 stops divided into 26 zones where buses of 500 bus lines are run. The longest length of the route of the bus line equals 29 and the shortest length equals 6 . The network is represented by graphs $G, G_{a}, G_{a r}$ and their parameters are described in Table 5.

The main goal of the experimental tests was to compare the computation time using the tested algorithms and it depends on the number of computed partial solutions, therefore it was counted in the tests. The second goal of the tests was to investigate an impact of the number of criterion functions on the computation time and the number of computed partial solutions. For this purpose, implemented algorithms have been modified to solve the BRP where only two criterion functions are used, i.e. the time and the cost of travel. In addition we have modified a record stored in the list $P S\left[v_{j}\right]$ and the record $\left(l_{j}, t_{s j}, c_{s j}, v_{i}, T_{i}, * P_{i}\right)$ was used. The modified record does not contain the list $L P$, instead, it contains the pointer $* P_{i}$ to the partial solution for the vertex $v_{i}$ and when the estimation is positive (line 17) we create a new record and add it to $P S\left[v_{j}\right]$. Thus, each path from $v_{s}$ to $v_{j}$ is represented by separate record in $P S\left[v_{j}\right]$. It increases the number of stored and analysed solutions. The goal of this modification was testing an impact of the form of the record representing the solution on the computation time and the number of computed partial solutions.

We have carried out 1371616 tests using the procedure FINDRoutes in both versions and 673719 tests using the Skriver algorithm, where two criterion functions are considered, and 220425 tests for version with three criterion functions. It was not possible to carry out the same number of tests for the Skriver algorithm as to the procedure FINDRouTES and the same number of tests for the both versions of Skriver algorithm due to out of memory. For the same reason, all tests have failed for the Brumbaugh-Smith algorithm, which determines all existing routes from $v_{s}$ to $v_{e}$. The aim of a single test was finding all routes belonging to the set of non-dominated solutions for the given pairs of vertices $v_{s}$ 
Table 6

The number of all computed non-dominated solutions using the FINDRoutes and the Skriver algorithms depending on the number of changes.

\begin{tabular}{|c|c|c|c|c|}
\hline \multirow{2}{*}{$\begin{array}{l}\text { Number of } \\
\text { changes }\end{array}$} & \multicolumn{2}{|c|}{ Criterion functions: $T, C$} & \multicolumn{2}{|c|}{ Criterion functions: $T, C, D$} \\
\hline & FindRoutes & Skriver & FindRoutes & Skriver \\
\hline 0 & 64771 & 61169 & 66060 & 53513 \\
\hline 1 & 553605 & 503427 & 596898 & 221679 \\
\hline 2 & 12217636 & 1771642 & 2783846 & 365116 \\
\hline 3 & 5774482 & 3136625 & 8509761 & 440584 \\
\hline 4 & 13311829 & 3328943 & 23268867 & 436417 \\
\hline 5 & 27485412 & 1405485 & 57994949 & 269344 \\
\hline 6 & 24613443 & 197354 & 112657304 & 197415 \\
\hline 7 & 2965135 & 41990 & 170024075 & 108229 \\
\hline 8 & 00521063 & 10783 & 275566765 & 62240 \\
\hline 9 & 195336 & 1086 & 362479435 & 10745 \\
\hline 10 & 65372 & 162 & 481644186 & 2308 \\
\hline 11 & 14350 & & 680149204 & \\
\hline 12 & & & 754155025 & \\
\hline 13 & & & 708535381 & \\
\hline 14 & & & 694029918 & \\
\hline 15 & & & 641701731 & \\
\hline 16 & & & 573463728 & \\
\hline 17 & & & 335616072 & \\
\hline 18 & & & 162101575 & \\
\hline 19 & & & 63804321 & \\
\hline 20 & & & 13519427 & \\
\hline 21 & & & 914334 & \\
\hline
\end{tabular}

and $v_{e}$ and the time of starting travel $T_{s}$. We present all results depending on the length of the route and the number of changes in the route.

The results of test experiments using the procedure FindRoutes and the Skriver algorithm have been presented in Table 6-11. We have shown the number of all computed non-dominated solutions during all experiments (Table 6 and Table 9), the maximal number of computed partial solutions during a single experiment (Table 7 and Table 10) and a maximal computation time in hrs:mins:secs format (Table 8 and Table 11). The results of tests using the procedure FindRoutes presented in Section 3 and the Skriver algorithm, where three criterion functions are considered, are denoted by "criterion functions: $T$, $C, D$ " and "criterion functions: $T, C$ " denotes results of tests using modified algorithms where only two criterion functions are considered. All records stored in the lists $P S\left[v_{j}\right]$ are treated as computed partial solutions. The results, where the partial solution is represented by a record containing the list $L P$, have been denoted by $L P$ and the results, where the modified record has been used, have been denoted by $* P$.

In both cases, i.e. solving the BRP where two and three criterion functions are used, the maximal number of partial solutions was computed using the Skriver algorithm. In the first case, it is about 419 times more than the maximal number of computed partial solutions by the procedure FindRoutes, where the modified record has been used and it is about 5295 times more if the record containing the list $L P$ was used. In the second case, these values are equal respectively to 315 and 1468 . The Skriver algorithm does 
Table 7

The maximal number of computed partial solutions in a single experiment using the FINDRoutes and the Skriver algorithms depending on the number of changes.

\begin{tabular}{|c|c|c|c|c|c|c|}
\hline \multirow{3}{*}{$\begin{array}{l}\text { Number } \\
\text { of } \\
\text { changes }\end{array}$} & \multicolumn{3}{|c|}{ Criterion functions: $T, C$} & \multicolumn{3}{|c|}{ Criterion functions: $T, C, D$} \\
\hline & \multicolumn{2}{|c|}{ FindRoutes } & \multirow[t]{2}{*}{ Skriver } & \multicolumn{2}{|c|}{ FindRoutes } & \multirow[t]{2}{*}{ Skriver } \\
\hline & $* P$ & $L P$ & & $* P$ & $L P$ & \\
\hline 0 & 1491 & 794 & 18957108 & 1073 & 814 & 43614321 \\
\hline 1 & 7582 & 2143 & 3011897 & 7015 & 2884 & 44384030 \\
\hline 2 & 19113 & 4217 & 47126308 & 19231 & 8233 & 44389232 \\
\hline 3 & 56434 & 5919 & 51243332 & 64751 & 16505 & 44389232 \\
\hline 4 & 71417 & 9211 & 52400859 & 74420 & 20146 & 44389232 \\
\hline 5 & 105666 & 9708 & 52546673 & 111912 & 26447 & 44384030 \\
\hline 6 & 107024 & 9924 & 52546673 & 140716 & 30240 & 44384030 \\
\hline 7 & 107024 & 9924 & 52546673 & 140716 & 30240 & 44244484 \\
\hline 8 & 107024 & 9924 & 51630198 & 140716 & 30240 & 44384030 \\
\hline 9 & 95361 & 9924 & 50734519 & 140716 & 30240 & 42006160 \\
\hline 10 & 105666 & 9924 & 694076 & 140716 & 30240 & 35940676 \\
\hline 11 & 73423 & 9924 & & 140716 & 30240 & \\
\hline 12 & 61973 & 8233 & & 140716 & 30240 & \\
\hline 13 & 24838 & 6281 & & 140716 & 30240 & \\
\hline 14 & & & & 140716 & 30240 & \\
\hline 15 & & & & 140716 & 29533 & \\
\hline 16 & & & & 140716 & 28261 & \\
\hline 17 & & & & 140385 & 26346 & \\
\hline 18 & & & & 137378 & 26511 & \\
\hline 19 & & & & 140716 & 26511 & \\
\hline 20 & & & & 140716 & 24793 & \\
\hline 21 & & & & 57428 & 21288 & \\
\hline
\end{tabular}

not apply conditions for estimation of the partial solutions that are applied by the procedure FindRoutes. Therefore, a larger space of solutions by the Skriver algorithm than by the procedure FINDRoutes is searched and the Skriver algorithm computes a larger number of partial solutions. For that reason, the Skriver algorithm has a larger memory complexity and it was not possible to carry out the same number of experiments as to the procedure FInDRoutes. The computation time depends on a searched space of solutions by the algorithm, therefore the computation time is larger for the Skriver algorithm.

With the increase of the length of the route and the number of changes, a searched space of solutions grows. For that reason, the number of computed partial solutions is growing with the increase of the number of changes and the length of the route. In the first case, i.e. in the BRP where two criterion functions are used, using the record with the list $L P$ in the procedure FindRoutes decreases about 10 times the number of computed partial solutions and it decreases the computation time. In the second case, the number of computed solutions is decreased 5 times.

Additionally, there was tested an influence of the number of criterion functions on the computation time and the number of computed partial solutions. Let us consider solution $P_{1}$ and $P_{2}$ for which $T\left(P_{1}\right)>T\left(P_{2}\right), C\left(P_{1}\right)>C\left(P_{2}\right)$ and $D\left(P_{1}\right)<D\left(P_{2}\right)$ are satisfied. In the first case, when only two criterion functions $T$ and $C$ are taken into account then $P_{2}$ dominates $P_{1}$. In the second case, when in addition the third criterion function $D$ is taken 
Table 8

The maximal computation time in a single experiment using the FindRoutes and the Skriver algorithms depending on the number of changes, the unit of the time is [hrs:mins:secs].

\begin{tabular}{|c|c|c|c|c|c|c|}
\hline \multirow{3}{*}{$\begin{array}{l}\text { Number } \\
\text { of } \\
\text { changes }\end{array}$} & \multicolumn{3}{|c|}{ Criterion functions: $T, C$} & \multicolumn{3}{|c|}{ Criterion functions: $T, C, D$} \\
\hline & \multicolumn{2}{|c|}{ FINDRoutes } & \multirow[t]{2}{*}{ Skriver } & \multicolumn{2}{|c|}{ FindRoutes } & \multirow[t]{2}{*}{ Skriver } \\
\hline & $* P$ & $L P$ & & $* P$ & $L P$ & \\
\hline 0 & 0:00:00 & 0:00:00 & $0: 02: 35$ & 0:00:00 & 0:00:00 & $0: 41: 30$ \\
\hline 1 & 0:00:00 & 0:00:00 & $0: 00: 14$ & 0:00:02 & 0:00:01 & $1: 19: 48$ \\
\hline 2 & 0:01:00 & 0:00:00 & $0: 59: 49$ & 0:00:15 & 0:00:07 & $1: 26: 29$ \\
\hline 3 & 0:00:00 & 0:00:00 & $3: 26: 19$ & $0: 01: 53$ & $0: 00: 57$ & $1: 26: 29$ \\
\hline 4 & 0:00:00 & 0:00:00 & $5: 44: 07$ & $0: 02: 24$ & 0:00:59 & $1: 26: 29$ \\
\hline 5 & 0:00:00 & 0:00:00 & $5: 44: 07$ & 0:02:33 & 0:00:59 & $1: 26: 29$ \\
\hline 6 & $0: 00: 32$ & 0:00:09 & $5: 44: 07$ & $0: 02: 43$ & 0:00:59 & $1: 26: 29$ \\
\hline 7 & 0:00:32 & 0:00:09 & $3: 51: 26$ & $0: 02: 43$ & 0:00:59 & 1:02:09 \\
\hline 8 & 0:00:06 & 0:00:04 & $3: 51: 26$ & $0: 02: 43$ & 0:00:59 & 1:09:44 \\
\hline 9 & 0:00:06 & 0:00:04 & $3: 51: 26$ & $0: 02: 43$ & 0:00:59 & 0:46:02 \\
\hline 10 & 0:00:00 & 0:00:00 & 0:00:04 & $0: 02: 43$ & 0:00:59 & $0: 18: 28$ \\
\hline 11 & 0:00:00 & 0:00:00 & & $0: 02: 43$ & 0:00:59 & \\
\hline 12 & 0:00:00 & 0:00:00 & & $0: 02: 43$ & 0:00:59 & \\
\hline 13 & 0:00:00 & 0:00:00 & & $0: 02: 43$ & 0:00:59 & \\
\hline 14 & & & & $0: 02: 43$ & 0:00:59 & \\
\hline 15 & & & & $0: 02: 43$ & 0:00:59 & \\
\hline 16 & & & & $0: 02: 43$ & 0:00:59 & \\
\hline 17 & & & & $0: 02: 32$ & 0:00:59 & \\
\hline 18 & & & & $0: 02: 24$ & $0: 00: 57$ & \\
\hline 19 & & & & $0: 02: 24$ & $0: 00: 57$ & \\
\hline 20 & & & & 0:02:11 & $0: 00: 51$ & \\
\hline 21 & & & & $0: 01: 52$ & 0:00:51 & \\
\hline
\end{tabular}

Table 9

The number of all computed non-dominated solutions using the FINDRoutes and the Skriver algorithms depending on the length of the route.

\begin{tabular}{|c|c|c|c|c|}
\hline \multirow{2}{*}{$\begin{array}{l}\text { Length of } \\
\text { the route }\end{array}$} & \multicolumn{2}{|c|}{ Criterion functions: $T, C$} & \multicolumn{2}{|c|}{ Criterion functions: $T, C, D$} \\
\hline & FindRoutes & Skriver & FindRoutes & Skriver \\
\hline $1-5$ & 153204 & 139158 & 173746 & 110206 \\
\hline $6-10$ & 517938 & 461066 & 1731971 & 552477 \\
\hline $11-15$ & 2217636 & 841915 & 7420803 & 919132 \\
\hline $16-20$ & 1740571 & 1265327 & 25055825 & 563973 \\
\hline $21-25$ & 2756929 & 1607326 & 85607310 & 21801 \\
\hline $26-30$ & 4075188 & 1649887 & 358860843 & 1 \\
\hline $31-35$ & 5503521 & 1539983 & 931244636 & \\
\hline $36-40$ & 7258514 & 1276342 & 2126130693 & \\
\hline $41-45$ & 9772586 & 905702 & 2165955723 & \\
\hline $46-50$ & 11845671 & 461975 & 399262866 & \\
\hline $51-55$ & 13029210 & 199495 & 15438041 & \\
\hline $56-60$ & 9471339 & 71627 & 4577491 & \\
\hline $61-65$ & 6262071 & 30175 & 1720131 & \\
\hline $66-70$ & 3096544 & 7763 & 355638 & \\
\hline $71-75$ & 1006427 & 890 & 43505 & \\
\hline $76-80$ & 249627 & 14 & 3434 & \\
\hline $81-85$ & 26952 & & 206 & \\
\hline 86-90 & 2226 & & & \\
\hline
\end{tabular}


Table 10

The maximal number of computed partial solutions in a single experiment using the FindRouTes and the Skriver algorithms depending on the length of the route.

\begin{tabular}{|c|c|c|c|c|c|c|}
\hline \multirow{3}{*}{$\begin{array}{l}\text { Length } \\
\text { of the } \\
\text { route }\end{array}$} & \multicolumn{3}{|c|}{ Criterion functions: $T, C$} & \multicolumn{3}{|c|}{ Criterion functions: $T, C, D$} \\
\hline & \multicolumn{2}{|c|}{ FindRoutes } & \multirow[t]{2}{*}{ Skriver } & \multicolumn{2}{|c|}{ FindRoutes } & \multirow[t]{2}{*}{ Skriver } \\
\hline & $* P$ & $L P$ & & $* P$ & $L P$ & \\
\hline $1-5$ & 1097 & 701 & 90169 & 764 & 611 & 341385 \\
\hline $6-10$ & 4229 & 1210 & 1496675 & 2681 & 1835 & 33140590 \\
\hline $11-15$ & 8801 & 2118 & 26578616 & 6544 & 3401 & 44169271 \\
\hline $16-20$ & 21273 & 3589 & 26578616 & 18174 & 7496 & 44389232 \\
\hline $21-25$ & 33918 & 5071 & 50138560 & 24757 & 10451 & 44389232 \\
\hline $26-30$ & 48616 & 7644 & 52400859 & 47360 & 18770 & 2050394 \\
\hline $31-35$ & 70023 & 9211 & 52546673 & 89688 & 24000 & \\
\hline $36-40$ & 88899 & 9708 & 52546673 & 137378 & 27774 & \\
\hline $41-45$ & 92482 & 9708 & 52546673 & 140716 & 30240 & \\
\hline $46-50$ & 95361 & 9924 & 52546673 & 140716 & 30240 & \\
\hline $51-55$ & 98962 & 9924 & 52546673 & 140716 & 30240 & \\
\hline $56-60$ & 107024 & 9924 & 52546673 & 140716 & 30240 & \\
\hline $61-65$ & 107024 & 9924 & 52546673 & 140716 & 30240 & \\
\hline $66-70$ & 107024 & 9924 & 49217090 & 140716 & 30240 & \\
\hline $71-75$ & 107024 & 9924 & 49217090 & 134626 & 30240 & \\
\hline $76-80$ & 84174 & 9924 & 6982760 & 83661 & 27959 & \\
\hline $81-85$ & 81987 & 9924 & & 79517 & 24352 & \\
\hline $86-90$ & 55883 & 7405 & & & & \\
\hline
\end{tabular}

Table 11

The maximal computation time in a single experiment using the FINDRoutes and the Skriver algorithms depending on the length of the route, the unit of the time is [hrs:mins:secs].

\begin{tabular}{|c|c|c|c|c|c|c|}
\hline \multirow{3}{*}{$\begin{array}{l}\text { Length } \\
\text { of the } \\
\text { route }\end{array}$} & \multicolumn{3}{|c|}{ Criterion functions: $T, C$} & \multicolumn{3}{|c|}{ Criterion functions: $T, C, D$} \\
\hline & \multicolumn{2}{|c|}{ FindRoutes } & \multirow[t]{2}{*}{ Skriver } & \multicolumn{2}{|c|}{ FIndRoutes } & \multirow[t]{2}{*}{ Skriver } \\
\hline & $* P$ & $L P$ & & $* P$ & $L P$ & \\
\hline $1-5$ & 0:00:00 & 0:00:00 & 0:00:00 & 0:00:00 & 0:00:00 & 0:00:11 \\
\hline $6-10$ & 0:00:00 & 0:00:00 & 0:00:06 & 0:00:00 & 0:00:00 & $0: 14: 57$ \\
\hline $11-15$ & 0:01:00 & 0:00:00 & $0: 04: 50$ & 0:00:01 & 0:00:00 & $0: 57: 26$ \\
\hline 16-20 & 0:00:00 & 0:00:00 & $0: 04: 50$ & 0:00:07 & 0:00:01 & $1: 26: 29$ \\
\hline $21-25$ & 0:00:00 & 0:00:00 & $3: 14: 12$ & 0:00:20 & 0:00:08 & $1: 26: 29$ \\
\hline $26-30$ & 0:00:00 & 0:00:00 & $3: 26: 19$ & $0: 02: 31$ & $0: 00: 54$ & \\
\hline $31-35$ & 0:00:00 & 0:00:00 & $3: 45: 59$ & $0: 02: 31$ & 0:00:59 & \\
\hline $36-40$ & 0:00:06 & 0:00:03 & $4: 39: 16$ & $0: 02: 31$ & 0:00:59 & \\
\hline $41-45$ & $0: 00: 32$ & 0:00:09 & $5: 44: 07$ & $0: 02: 43$ & 0:00:59 & \\
\hline $46-50$ & 0:00:32 & 0:00:09 & $5: 44: 07$ & $0: 02: 43$ & 0:00:59 & \\
\hline $51-55$ & 0:00:32 & 0:00:09 & $4: 39: 16$ & $0: 02: 43$ & 0:00:59 & \\
\hline $56-60$ & $0: 00: 32$ & 0:00:09 & $3: 51: 26$ & $0: 02: 43$ & $0: 00: 59$ & \\
\hline $61-65$ & 0:00:32 & 0:00:09 & $3: 51: 26$ & $0: 02: 43$ & 0:00:59 & \\
\hline $66-70$ & 0:00:01 & 0:00:01 & $3: 51: 26$ & $0: 02: 24$ & 0:00:59 & \\
\hline $71-75$ & 0:00:01 & 0:00:01 & $3: 51: 26$ & $0: 02: 32$ & $0: 00: 54$ & \\
\hline $76-80$ & 0:00:00 & 0:00:00 & $0: 01: 57$ & 0:02:06 & 0:00:43 & \\
\hline $81-85$ & 0:00:00 & 0:00:00 & & 0:00:08 & 0:00:07 & \\
\hline $86-90$ & 0:00:00 & 0:00:00 & & & & \\
\hline
\end{tabular}


into account then $P_{1}$ and $P_{2}$ are non-dominated solutions. For that reason, the number of computed partial solution in the second case is greater than in the first and it increases the computation time. This was the reason why the different number of tests was carried out by the Skriver algorithm in both cases. It was not possible to carry out the same number of tests due to out of memory. Adding the third criterion function increases about 3 times the number of computed partial solutions by the procedure FindRoutes where the record with the list $L P$ is used. For the second version of the procedure it increases the number of computed partial solutions about 1.5 times.

The number of criterion functions increases the number of non-dominated solutions. In the first case, i.e. in the BRP where two criterion functions are used, the maximal number of non-dominated solutions in the single test was 461440 but only 385 solutions differ from each other in the path, other solutions were the same path and differ from each other only in the times of departure. In the second case, these values are equal 3162693 and 66 respectively. Thus adding the length of the route as the third criterion function increases about 7 times the number of non-dominated solutions.

\section{Conclusions}

In this paper we considered the bus routing problem (BRP), in particular, we analysed the problem and properties of a route. The goal of the problem is to find a route from the given start stop to the given final stop minimizing the time and the cost of travel and the length of the route, where the time of starting travel at the start stop is given additionally. The $\mathrm{BRP}$ is an example of the multicriteria shortest path (MSP) problem with variable weights which the solution is the set of non-dominated solutions. The MSP problem has already been considered in the literature and several algorithms have been proposed, however, these algorithms assume constant weights. We have analysed differences between the MSP problem with variable and constant weights. Additionally, the results of solving the BRP where the goal is to minimize only the time and the cost of travel are presented.

In the paper we proposed a label correcting algorithm with storing partial solutions for solving the BRP. The algorithm makes it possible to find all routes belonging to the set of non-dominated solutions. As shown, the MSP is known to be NP-complete and in the worst case, the number of non-dominated solutions grows exponentially with the $n$ value. The pessimistic time complexity of the algorithm is determined by the maximal number of non-dominated solutions and it equals $O\left(d^{n}\right)$, where $n$ equals a number of vertices in the graph representing the bus network and $g$ equals a maximum out-degree of a vertex in the graph.

On the basis of the results of experimental tests we found that presented algorithm exhibits a reasonable execution time for the bus network containing about 1200 stops. Additionally, the results demonstrate that the number of non-dominated solutions is not exponential in practice. In the algorithm we take into consideration only three criterion functions representing the time and the cost of travel and the length of the route but it is possible to add other criterion functions. 


\section{References}

Azevedo, J.A., Martins, E.Q.V. (1991). An algorithm for the multiobjective shortest path problem on acyclic networks. Investigação Operacional, 11(1), 52-69.

Bellman, R. (1958). On a routing problem. Quarterly of Applied Mathematics, 16(1), 87-90.

Brumbaugh-Smith, J., Shier, D. (1989). An empirical investigation of some bicriterion shortest path algorithms. European Journal of Operational Research, 43(2), 216-224.

Chartrand, G., Lesniak, L., Zhang, P. (2010). Graphs \& Digraphs. 5th edition, Chapman \& Hall/CRC, Boca Raton.

Climaco, J.C. Martins, E.Q.V. (1982). A bicriterion shortest path algorithm. European Journal of Operational Research, 11(4), 399-404.

Coello Coello, C.A. van Veldhuizen, D.A. Lamont, G.B. (2002). Evolutionary Algorithms for Solving MultiObjective Problems. Kluwer Academic, New York.

Corley, H.W., Moon, I.D. (1985). Shortest paths in networks with vector weights. Journal of Optimization Theory and Application, 46(1), 79-86.

Daellenbach, H.G., De Kluyver, C.A. (1980). Note on multiple objective dynamic programming. Journal of the Operational Research Society, 31(7), 591-594.

Dell'Olmo, P., Gentili, M., Scozzari, A. (2005). On finding dissimilar Pareto-optimal paths. European Journal of Operational Research, 162(1), 70-82.

Dijkstra, E.W. (1959). A note on two problems in connexion with graphs. Numerical Mathematics, 1, $269-271$.

Ehrgott, M. (1999). Multicriteria optimization. Vorlesungsskripte, Sonstiges. Department of Mathematics, University of Kaiserslautern, Germany.

Ehrgott, M., Gandibleux, X. (1999). A survey and annotated bibliography of multiobjective combinatorial optimization. OR Spektrum, 22(4), 425-460.

Floyd, R. (1962). Algorithm 97: Shortest path. Communications of the ACM, 5(6), 345.

Ford, L.R. (1956). Network Flow Theory. Report P-923, The Rand Corporation, Santa Monica.

Garey, M., Johnson, D. (1990). Computers and Intractibility: A Guide to the Theory of NP-Completeness. Freeman, New York.

Hansen, P. (1980). Bicriterion path problems. Multiple Criteria Decision Making: Theory and Application, Springer, Berlin, pp. 109-127.

Henig, M.I. (1985). The shortest path problem with two objective functions. European Journal of Operational Research, 25(2), 281-291.

Johnson, D.B. (1977). Efficient algorithms for shortest paths in sparse networks. Journal of the ACM, 24(1), $1-13$.

Jungnickel, D. (1999). Graphs, Networks and Algorithms. 2nd edition, Springer, Berlin.

Korhonen, P. (1992). Multiple criteria decision support - a review. European Journal of Operational Research, 63(3), 361-375.

Machuca, E., Mandow, L., Pérez de la Cruz, J.L. (2009). An evaluation of heuristic functions for bicriterion shortest path problems. In: Proceedings of the XIV Portuguese Conference on Artificial Inteligence (EPIA 2009), Universidade de Aveiro, Portugal, pp. 205-216.

Mandow, L., Pérez-de-la-Cruz, J.L. (2008a). Frontier search for bicriterion shortest path problems. In: Proceeding of the 2008 Conference on ECAI 2008 18th European Conference on Artificial Intelligence, pp. 480-484.

Mandow, L., Pérez-de-la-Cruz, J.L. (2008b). Path recovery in frontier search for multiobjective shortest path problems. Journal of Intelligent Manufacturing, 21(1), 89-99.

Martí, R., González Velarde, J.L., Duarte, A. (2009). Heuristics for the bi-objective path dissimilarity problem. Computers \& Operations Research, 36(11), 2905-2912.

Martins, E.Q.V. (1984). On a multicriteria shortest path problem. European Journal of Operational Research, 16(2), 236-245.

Martins, E.Q.V., Pascoal, M.M.B., Rasteiro, D.M.L.D., Santos. J.L.E. (1999). The optimal path problem. Investigação Operacional, 19, 43-60.

Mote, J., Murthy, I., Olson, D.L. (1991). A parametric approach to solving bicriterion shortest path problems. European Journal of Operational Research, 53(1), 81-82.

Pareto, V. (1896). Course d'Economie Politique. Rouge, Lausanne.

Raith, A., Ehrgott, M. (2009). A comparison of solution strategies for biobjective shortest path problems. Journal Computers and Operations Research, 36(4), 1299-1331. 
Roy, B., Vincke, P. (1981). Multicriteria analysis: survey and new directions. European Journal of Operational Research, 8(3), 207-218.

Skriver, A.J.V., Andersen, K.A. (2000a). A classification of bicriteria shortest path (BSP) algorithms. AsiaPacific Journal of Operational Research, 17, 199-212.

Skriver, A.J.V., Andersen, K.A. (2000b). A label correcting approach for solving bicriterion shortest-path problems. Computers \& Operations Research, 27(6), 507-524.

Tung, C.T., Chew, K.L. (1988). A bicriterion Pareto-optimal path algorithm. Asia-Pacific Journal of Operational Research, 5, 166-172.

Tung, C.T., Chew, K.L. (1992). A bicriterion Pareto-optimal path algorithm. European Journal of Operational Research, 62(2), 203-209.

Ulungu, E.L., Teghem, J. (1994). Multi-objective combinatorial optimization problems: a survey. Journal of Multi-Criteria Decision Analysis, 3(2), 83-104.

Voorneveld, M. (2003). Characterization of Pareto dominance. Operations Research Letters, 31(1), 7-11.

Warshall, S. (1962). A theorem on Boolean matrices. Journal of the ACM, 9(1), 11-12.

Wilson, R.J. (1996). Introduction to Graph Theory. 4th edition, Addison-Wesley, Reading.

J. Widuch was awarded the doctor of engineering sciences degree at the Silesian University of Technology, Institute of Informatics in 2008. At present works as an assistant professor in the Institute of Informatics of Silesian University of Technology. His main research interest is connected with multicriteria optimization algorithms in transportation problems and parallel computing. He is a member of Upper Silesian Regional Committee of Polish Olympiad in Informatics.

\section{Autobusų maršrutų sudarymo algoritmas pagrịstas žymeklio korega- vimu ir dalinių sprendinių įsiminimu}

\section{Jacek WIDUCH}

Straipsnyje aprašytas autobusų maršrutų sudarymo (BRP) algoritmas, kurio tikslas surasti maršrutą nuo pradinès iki galinès stotelès minimizuojant laiką, kelionès kainą ir ilgi. Kelionès pradžios laikas pradinèje stotelèje yra duotas. Išanalizavus uždavinị pateiktos maršrutu savybès. BRP yra daugiakriterès optimizacijos uždavinys, kurio sprendiniais yra nedominuojami sprendiniai. Šiame straipsnyje pasiūlytas žymeklio koregavimo algoritmas išsaugantis dalinius sprendinius. Algoritmu randami visi maršrutai priklausantys nedominuojamų sprendinių aibei. Taip pat pateikti eksperimentinio testavimo rezultatai. Gauti rezultatai palyginti su BRP użdavinio sprendimo rezultatais, kai minimizuojamas tik laikas, ar tik kaina, ar tik maršruto ilgis. 